\title{
Author reply: No immediate increase in morbidity of infants delivered by term cesarean section for placenta previa: some concerns
}

\author{
Asnat Walfisch $^{1} \cdot$ Eyal Sheiner $^{1}$
}

Received: 1 March 2016/Accepted: 2 March 2016/Published online: 25 March 2016

(C) Springer-Verlag Berlin Heidelberg 2016

We appreciate Dr. Matsubara and his co-authors' interest in our study [1]. Indeed, neonatal anemia is a concern in placenta previa newborns and may be influenced by the surgical technique used $[2,3]$. Our study was not designed to assess neonatal anemia, which may or may not have been more prevalent in the placenta previa cases. Nevertheless, Apgar scores and perinatal mortality were comparable, suggesting that this was probably not a significant feature. Our point was mainly to direct attention to gestational age at delivery in these cases, as the major determinant of neonatal outcome rather than the placenta previa per se.

On the other hand, there is no doubt with regard to increased maternal hemorrhagic morbidity surrounding placenta previa pregnancies, during the pregnancy course and, dramatically so, during the cesarean delivery itself [4, 5]. We could not agree more with regard to the need of a specialized and experienced obstetrical team to insure maximal maternal safety. Although not necessarily always justified, it is routine in our hospital, as in many others, to have a midwife and a neonatologist present in all cesarean deliveries; thus, we did not focus our attention on this issue. However, the question raised by Dr. Matsubara et al. is indeed important, as we acknowledge the fact that the presence of a neonatologist is not a uniform protocol. We feel that our study provides some reassuring data to the management of term placenta previa newborns and should stimulate further studies and reassessment of management protocols.

\section{Compliance with ethical standards}

conflict of interest The authors report no conflict of intrest.

\section{References}

1. Walfisch A, Sheiner E (2016) Placenta previa and immediate outcome of the term offspring. Arch Gynecol Obstet (Epub ahead of print)

2. Schneiderman M, Balayla J (2013) A comparative study of neonatal outcomes in placenta previa versus cesarean for other indication at term. J Matern Fetal Neonatal Med 26(11):1121-1127

3. Matsubara S, Baba Y, Ohkuchi A (2015) Cesarean incision in case of placenta previa: does the transplacental approach cause fetal anemia? Acta Obstet Gynecol Scand 94(2):226-227

4. Rosenberg T, Pariente G, Sergienko R, Wiznitzer A, Sheiner E (2011) Critical analysis of risk factors and outcome of placenta previa. Arch Gynecol Obstet 284(1):47-51

5. Sheiner E, Shoham-Vardi I, Hallak M, Hershkowitz R, Katz M, Mazor M (2001) Placenta previa: obstetric risk factors and pregnancy outcome. J Matern Fetal Neonatal Med 10(6):414-419

Asnat Walfisch

asnatwalfisch@yahoo.com

1 Department of Obstetrics and Gynecology, Soroka University Medical Center, Beer-Sheva, Israel 